\title{
A review of factors limiting physical activity among young children from low-income families
}

\author{
Seung Ho Chang', Kijeong Kim²,* \\ 'Department of Kinesiology, San Jose State University, San Jose, CA, USA \\ ${ }^{2}$ School of Exercise \& Sport Science, College of Natural Sciences, University of Ulsan, Ulsan, Korea
}

Children need to participate in structured physical activities to avoid obesity and to engage in sports and games in the future. However, certain groups of our population have little access to enhance levels of physical activity. One such group is children from low-income families who are more likely to have less opportunity for participation in physical activity because of factors such as unsafe streets or limited access to playgrounds. According to the United States Department of Health and Human Services, there is a strong relationship between family income and physical activity with low-income families being the most sedentary. In order to design effective physical activity interventions for those children, there is a need to identify factors influencing physical activity in these populations, yet, there has been limited research that explores this issue. Therefore, the purpose of this article was to review the literature on factors limiting physical activity among low-income children.

Keywords: Physical activity, Child, Low-income family, Preschoolers

\section{INTRODUCTION}

Childhood obesity is a significant public health issue in the United States and worldwide. In 2006, the prevalence of obesity increased from $6.5 \%$ in 1980 to $19.6 \%$ among 6 through 11 years old children, while the prevalence of obesity for children age 12 through 17 years old increased from 5\% to $18.1 \%$ during that same time span (Centers for Disease Control and Prevention [CDC], 2010). In addition, the obesity rate among preschool children age 2 through 5 years old increased from $5.0 \%$ to $10.4 \%$ between 1976-1980 and 2007-2008 (CDC, 2010). Regular physical activity in children is one of the major recommendations to prevent or reduce the incidence of childhood obesity. Since overweight is caused by an energy imbalance of more calories consumed than used, physical activity can play a key factor in maintaining an appropriate energy balance because it helps burn calories that are consumed in excess. For most children in the United States, free time is spent engaging in physical activity. However, research by Rideout and Hamel (2006) has shown a steady decline in children's free time by 25\% between 1981 and 1997 due to an increase in the amount of time children spend in structured activities (i.e., school lessons or organized sports) which has contributed to higher amounts of inactivity within the culture of U.S. communities. Experts recommend that increasing physical activity is significant to prevent the continued onset of overweight and obesity among preschool children (CDC, 2006). Although people know the importance of physical activity to prevent childhood obesity including other diseases, segments of our population have little access to health enhancing levels of physical activity. One such group is children from low-income families who are more likely to have less opportunity for participation in physical activity. According to the United States Department of Health and Human Services there is a strong relationship between family income and physical activity with low-income families being the most sedentary (U.S. Department of Health and Human Services, 1996). Furthermore, several studies (DuRant et al., 1994; Lindsay et al., 2006; Powell et al., 2003) have found that many families and children are inactive at home and are likely to engage in sed-
${ }^{*}$ Corresponding author: Kijeong Kim (D) https://orcid.org/0000-0001-9233-3096 School of Exercise \& Sport Science, College of Natural Sciences, University of Ulsan, 93 Daehak-ro, Nam-gu, Ulsan 44610, Korea

Tel: +82-52-259-2381, Fax: +82-52-259-1696, E-mail: kijeongk@ulsan.ac.kr

Received: July 3, 2017 / Accepted: August 7, 2017
This is an Open Access article distributed under the terms of the Creative Commons Attribution Non-Commercial License (http://creativecommons.org/licenses/by-nc/4.0/) which permits unrestricted non-commercial use, distribution, and reproduction in any medium, provided the original work is properly cited. 
entary behaviors like playing video games, watching TV, and computer activities. In order to maintain adequate levels of health among children adolescences, there is a need to promote the engagement of physical activity for children from low-income families. Therefore, the purpose of this article was to review studies focused on physical activity for children from low income families and identify factors related to their lack of physical activity.

\section{FACTORS LIMITING PHYSICAL ACTIVITY}

\section{Home environment}

Goodway and Smith (2005) investigated the environmental factors influencing the physical activity of urban African American children from low income families. They interviewed 59 young African American children and their mothers or primary female caregivers living in low-income urban neighborhoods. The results of study reported that adult interactions and encouragement of physical activity have influenced young children's behaviors of physical activity. Furthermore, children stated that mothers were not normally involved in the play of their preschool-aged children and mothers have preferred sedentary and quiet activities such as watching television or playing video games. Furthermore, lack of access to safe and appropriate spaces to do outdoor plays, unsafe neighborhoods and no organized affordable physical activity programs were identified as the barriers to physical activity for young children. Lindsay et al. (2006) investigated evidences on the importance of parents helping their children to develop healthy eating and physical activity behaviors throughout various developmental stages such as infancy, preschool, and school age. Participants in this study were low income Latino mothers of young children. Lindsay et al. (2006) found that parents play a critical role at home and are essential in preventing childhood overweight and obesity, as they should focus on understanding their role in the child's dietary habits, physical activity and sedentary behaviors. Furthermore, lack of social support for physical activity, parents' needs (i.e., work), patterns of life (i.e., sedentary or active life style), the safety of neighborhood and seasonal factors such as cold or hot weather emerged as the factor influencing physical activities.

\section{Accessibility and resources}

Rich et al. (2005) interviewed 76 mothers or caregivers of Latino children receiving the special supplemental nutrition program for women, infants, and children. $48 \%$ of participants identified an indoor context as the safe place for play. In addition, Goodway and Smith (2005) and Lindsay et al. (2009) identified barriers to physical activity in indoor settings, particularly for children living in apartment buildings. Some mothers reported that they forced children to hold in sedentary quiet activities (i.e., screen time activities) so that neighbors would not complain about the noise. Williams et al. (2009) piloted a program called "Animal Trackers" for preschool children. This program included a 10-unit curriculum intended to promote structured physical activity, gross motor skills and teacher friendly physical activity program in preschool settings. The results of this study showed that and the curriculum averaged approximately $47 \mathrm{~min}$ of structured physical activity per week across the 10-week intervention period received positive ratings from the teachers. This study recommended that organized and structured programs should be provided for children from low-income families to promote their levels of physical activity. Bosch and Reicks (2000) have conducted interviews to investigate the eating and physical activity behaviors of low-income families with young children. The study found that barriers to being active with young children were lack of time, energy, and limited resources. Furthermore, the results of study highlighted that families need to adjust daily routine tasks to meet physical activity needs and regular physical activities with minimal equipment need to be implemented at home. Goodway and Smith (2005) suggested that young children from low-income families may have less chance to engage in physical activity because mothers of African American children living in low-income urban areas have a lack of time and energy for supporting and participating in the physical activity with their young child. Similarly, Lindsay et al. (2009) stated that Latina mothers living in low-income urban communities with young children reported that it is hard to supervise children in outdoor play due to their own work schedules and domestic responsibilities. In addition, Stoffel and Berg (2008) found that access to material resources was related to a barrier to participation in a variety of activities (including physical play activities) for young children from low-income.

\section{CONCLUSIONS}

Studies (Goodway and Smith, 2005; Lindsay et al., 2009) aiming to understand the relationship between home environmental factors and physical activity with children suggested that parent's beliefs and engagement in physical activity with young children may influence young children's physical activity behaviors. Both studies found that beliefs related to adult engagement in physical activity were different for low-income African American and Lati- 
na mothers because of culturally different beliefs and values of physical activity parent has. Children living in low-income urban neighborhoods are hard to get opportunities to participant in physical activity due to neighborhood safety such as unsafe streets and limited access to safe playgrounds (Goodway and Smith, 2005; Lindsay et al., 2009). In addition, increased amount of time in indoor setting are also one of the barriers to decrease physical activity with young children (Rich et al., 2005). Finally, children from low-income family often have parents with limited financial resources to be able to afford physical activity opportunities for their children. Furthermore, parental work, school, or other caretaking demands limit the time that parents can interact with their children in activity related modeling. In conclusion, there have been a limited number of studies and inconsistent findings have been reported. More studies need to be conducted to examine the relationship various factors and physical activity for young children from low-income families.

\section{CONFLICT OF INTEREST}

No potential conflict of interest relevant to this article was reported.

\section{ACKNOWLEDGMENTS}

This work was supported by the 2017 Research Fund of University of Ulsan.

\section{REFERENCES}

Bosch A, Reicks M. Physical activity for preschool children: growing up fit - together. J Nutr Educ Behav 2000;32:64-65.

Centers for Disease Control and Prevention. Prevalence of obesity among children and adolescents: United States, Trends 1963-1965 Through 2007-2008 [Internet]. Atlanta (GA): Centers for Disease Control and Prevention; 2010 Jun [updated 2010 June 4; cited 2017 Jul 27]. Available from: https://cdc.gov/nchs/data/hestat/obesity_child_07_08/obesity_child_07_08.htm.
Centers for Disease Control and Prevention. Prevalence of overweight among children and adolescents: United States, 2003-2004 [Internet]. Atlanta (GA): Centers for Disease Control and Prevention; [2006] [updated 2015 Nov 6; cited 2017 Jul 27]. Available from: http://www.cdc. gov/nchs/data/hestat/overweight/overweight_child_03.htm.

DuRant RH, Baranowski T, Johnson M, Thompson WO. The relationship among television watching, physical activity, and body composition of young children. Pediatrics 1994;94(4 Pt 1):449-455.

Goodway JD, Smith DW. Keeping all children healthy: challenges to leading an active lifestyle for preschool children qualifying for at-risk programs. Fam Community Health 2005;28:142-155.

Lindsay AC, Sussner KM, Greaney ML, Peterson KE. Influence of social context on eating, physical activity, and sedentary behaviors of Latina mothers and their preschool-age children. Health Educ Behav 2009;36: 81-96.

Lindsay AC, Sussner KM, Kim J, Gortmaker S. The role of parents in preventing childhood obesity. Future Child 2006;16:169-186.

Powell KE, Martin LM, Chowdhury PP. Places to walk: convenience and regular physical activity. Am J Public Health 2003;93:1519-1521.

Rich SS, DiMarco NM, Huettig C, Essery EV, Andersson E, Sanborn CF. Perceptions of health status and play activities in parents of overweight Hispanic toddlers and preschoolers. Fam Community Health 2005;28:130-141.

Rideout VJ, Hamel E. The media family: electronic media in the lives of infants, toddlers, preschoolers and their parents. Menlo Park (CA): Henry J. Kaiser Family Foundation; 2006.

Stoffel A, Berg C. Spanish translation and validation of the Preschool Activity Card Sort. Phys Occup Ther Pediatr 2008;28:171-189.

U.S. Department of Health and Human Services. Physical activity and health: a report of the surgeon general [Internet]. Atlanta (GA): U.S. Department of Health and Human Services (USDHHS), Centers for Disease Control and Prevention, National Center for Chronic Disease Prevention and Health Promotion; 1996 [cited 2017 July 27]. Available from: https://www.cdc.gov/nccdphp/sgr/pdf/sgrfull.pdf.

Williams CL, Carter BJ, Kibbe DL, Dennison D. Increasing physical activity in preschool: a pilot study to evaluate animal trackers. J Nutr Educ Behav 2009;41:47-52. 